\title{
Few Results on Total Graph of Complete Graph
}

\author{
Ancy K. Joseph \\ College of Engineering, AMA International University, Bahrain
}

\begin{abstract}
Let $K_{n}$ be a complete graph with $n$ vertices and $T\left(K_{n}\right)$ be the total graph of a complete graph $K_{n}$. Through this paper it has been proved that $T\left(K_{n}\right)$ is a strongly regular graph. Also, a general formula has been derived to calculate the four parameter $(n, k, \lambda, \mu)$ of strongly regular graph, $T\left(K_{n}\right)$. Further, eigen values, spectrum and energy of the graph $T\left(K_{n}\right)$ has been found.
\end{abstract}

Keywords: Total Graph, Complete Graph, Regular Graph, Strongly Regular, Spectrum, Energy

\section{INTRODUCTION}

Graph theory is a branch of mathematics which deals with vertices and edges. Edges connecting the vertices. Graphs are ever-present miniature of both from nature and man-made structures. Graphs are the miniature of physical problems it can handle changes in the real world which is dynamic. The subject Graph Theory had its beginnings in recreational problem, but now it has evolved into a major area of mathematical research work with application in various fields like chemistry, operation research, social science, computer science, logistics etc. The concept of graph was first mentioned by Euler. The actual problem that he was considering can be regarded as flippant puzzle; which arose from the physical world. After a century of a paper by Euler on the famous Konigsberg bridge problem. Topology was introduced by listing, Cayley was studying a specific analytical form that arose from differential calculus to learn a specific type of graphs, which is called the trees. From the above almost frivolous beginning has grown modern graph theory, which now embraces topics such as domination, neighbourhood, algebraic graph theory, fractional graph theory, switching, design theory, to name just a few. All topics deal essentially with abstract concepts, although many have potential application in practical.

Various studies have been carried out on strongly regular graphs. Few relationships among the parameters are established. Different types of graphs are taken and their parameters such as $(n, k, \lambda, \mu)$ are calculated. As we know that adjacency, matrix, represents the non-pictorial form of corresponding graph and also many researchers concluded that from adjacency matrix one can calculate the Eigen values of the corresponding graph. An application of graph theory in chemistry was started by Erich Huckel in 1930's in his study of molecular orbital theory. A frame of non-saturated hydrocarbon in quantum chemistry, was structured by graphs and was converted to a graph theory problem. The level of energy of electrons in molecules of such non-saturated hydrocarbon were surprisingly Eigen values of the corresponding graphs and hence the name was coined relating to it.

\subsection{Objectives:}

Objectives of the work is to study the total graph of a complete graph. Also prove that total graph of a complete graph is strongly regular. Eigen values of the graph obtained by graph operation is found and hence the spectrum. These values have been used to calculate the energy of graph. It is purely in the interest of mathematical aspect. "

\subsection{Scope and Limitation}

Total graph of a complete graph has not been studied much. Also, there are various examples on strongly regular graph parameters but not total graph of a complete graph. Energy of graphs is essentially an abstract concept, although it has many potential applications in practical. The results derived can be used in chemistry and other fields. The significance of graph operation its spectrum and energy lies when it could be transformed to a real-time problem and the parameters and related results answers the real-time problems.

\section{LITERATURE REVIEW}

In 1936, D. Konig [12], the Hungarian Mathematician wrote the first book on graph theory. Later book on graph theory also written by C. Berge [2], O. Ore[13] and F. Harary[7]. Application of graph theory with different branches like engineering technology, biological science, archeology, ecology, planning etc can be found in the book of F.S Robestx. The connection between graph theory with other branches of mathematics were discussed in the book of L.U Beincles and R.J Wilson. 


\section{International Advanced Research Journal in Science, Engineering and Technology}

Vol. 6, Issue 3, March 2019

In [5] D. F. Anderson and Badawi introduced the total graph. Strongly regular graph was introduced by Raj Chandra Bose in 1963 [9]. It has been extended over years and finds its relation in various structures. A graph is strongly regular if the adjacency matrix yields three eigen values [10]. These graphs have various spectral properties. There are some unsolved problems as well. Most of the strongly regular graphs are asymmetric.

The total pie-electron energy $\mathrm{E}(\mathrm{G})$, created lots of curiosity among the researchers after C.A Coulson [4] initiated his work on it. I. Gutman [13] in 1978, for the first time brought forth the concepts of energy of graph. He defined it as addition of absolute value of Eigen values of a graph. Due to the great success of graph energy concept few analogous quantities such as Laplacian energy, incidence energy, distance energy, skew energy and soon were also conceived. For more detailed survey, see $[1,6,11]$

\section{RESEARCH METHODOLOGY}

\subsection{Formation of total graph from complete graph}

Two kinds of graph discussed here is Complete graph and total graph (which is graph obtained by operations) We know that a complete graph denoted by $\mathbf{K}_{\mathbf{n}}$ is a graph where every pair of distinct vertices is connected by a unique edge. The graph considered here is simple (no loops and multiple edges) and is undirected graph. Total graph denoted by $\mathrm{T}(\mathrm{G})$ of a graph $\mathrm{G}$ is a graph in which the set of vertices is both the set of vertices and edge set of $\mathrm{G}$ and any two vertices in $\mathrm{T}(\mathrm{G})$ are said to be adjacent if and only if their corresponding elements are either adjacent or incident in $\mathrm{G}$.

Total graph of a complete graph is denoted by $\mathrm{T}\left(\mathbf{K}_{\mathbf{n}}\right)$ and the adjacency matrix of $\mathrm{T}\left(\mathbf{K}_{\mathbf{n}}\right)$ is denoted by $\mathrm{A}\left(\mathrm{T}\left(\mathbf{K}_{\mathbf{n}}\right)\right)$.

Lemma 3.1.1. Vertices of the total graph obtained from the complete graph is $\frac{n(n+1)}{2}$.

Proof: A complete graph has $n$ vertices and $\frac{n(n-1)}{2}$ edges. From the definition of total graph of complete graph, the vertices of $T\left(K_{n}\right)$ is the sum of vertices and edges of complete graph. Therefore, total graph has $\left(n+\frac{n(n-1)}{2}\right)$ vertices. ie, $\frac{\mathrm{n}(\mathrm{n}+1)}{2}$ vertices.

Lemma 3.1.2. Total graph of a complete graph is $2(n-1)$ regular.

Proof: One vertex $v_{1}$ say in $k_{n}$ will be adjacent to $(n-1)$ vertices and hence there will be $(n-1)$ edges connecting these vertices with $v_{1}$. Hence in $T\left(K_{n}\right),(n-1)+(n-1)=2(n-1)$ will be the degree of $v_{1}$. Hence the graph is $2(n-1)$ regular.

Lemma 3.1.3. Edges of total graph of a complete graph is $\frac{n\left(n^{2}-1\right)}{2}$.

Proof: From Lemma 3.1.2, total graph of a complete graph is $2(n-1)$ regular. Hence there are $2(n-1)$ edges incident on each vertex of total graph of a complete graph and there are $\frac{n(n+1)}{2}$ vertices (from Lemma 3.1.1) Hence the sum of the degrees of all the vertices are $2(n-1) X \frac{n(n+1)}{2}$. Also we know that an edge contributes to the degree of a vertex twice. Therefore, the total number of edges is $\frac{2(n-1) \times \frac{n(n+1)}{2}}{2}$. Hence there are $\frac{n\left(n^{2}-1\right)}{2}$ edges.

\subsection{Total graph of a complete graph is Strongly regular graph}

A graph $G$ of order $n$ is called strongly regular with parameters $(n, k, \lambda, \mu)$ if

- $\quad$ every vertex of $\mathrm{G}$ has degree $\mathrm{k}$,

- if $u$ and $v$ are adjacent vertices of $G$, then the number of common neighbors of $u$ and $v$ is $\lambda$,

- if $u$ and $v$ are non-adjacent vertices of $G$, then the number of common neighbors of $u$ and $v$ is $\mu$

Lemma 3.2.1. In a total graph of a complete graph with $n>2$, the number of common neighbours for any two adjacent vertices is $n-1$.

Proof: Let total graph of a complete graph be $\mathrm{T}\left(\mathrm{k}_{\mathrm{n}}\right)$ where $\mathrm{n}>2$. We consider here two cases Case1: Vertex to vertex adjacency

Two vertex say $u$ and $v$ are adjacent in $T\left(k_{n}\right)$ if they are adjacent in $k_{n}$. A vertex say $u$ in $k_{n}$ is adjacent to $n-1$ vertices and so is $v$. Therefore, the two vertices are adjacent to $(n-2)$ vertices. Also the edge $\left(e_{1}\right)$ connecting $u$ and $v$ 


\section{International Advanced Research Journal in Science, Engineering and Technology}

Vol. 6, Issue 3, March 2019

in $k_{n}$ is a vertex in $T\left(k_{n}\right)$. Hence $u$ and $v$ are adjacent to $e_{1}$. No other vertices in $T\left(k_{n}\right)$ which were previously edges in $\mathrm{k}_{\mathrm{n}}$ are adjacent to both $\mathrm{u}$ and $\mathrm{v}$ as this graph forms triangles with vertex. This makes it $(\mathrm{n}-2)+1$ ie, $(\mathrm{n}-1)$ adjacent vertices.

Case2: Vertex to edge adjacency

Consider a vertex $u$ in $k_{n}$ and an edge say $e_{1}$ incident on it. We know that $u$ and $e_{1}$ are vertices in $T\left(k_{n}\right)$. One end of edge $e_{1}$ is $u$ let the other vertex be $v$ in $k_{n}$. Hence $u$ and $e_{1}$ are adjacent or incident to $v$. Degree of the vertex $u$ in $k_{n}$ is $(n-1)$. Excluding the $e_{1}$, it makes $(n-2)$. All these $(n-2)$ edges are vertices in $T\left(k_{n}\right)$. Therefore $u$ and $e_{1}$ are adjacent to these $(n-2)$ vertices in $T\left(k_{n}\right)$ and $v$. Therefore, $u$ and $e_{1}$ has $(n-1)$ adjacent vertices in $T\left(k_{n}\right)$. Same with other vertices.

From Case 1 and Case 2 we can conclude that in a total graph of a complete graph the number of common neighbours for any two adjacent vertices is $n-1$.

Lemma 3.2.2. In a total graph of a complete graph with $n>2$, the number of common neighbours for any two nonadjacent vertices is 4 .

Proof : Here there is only one case. Vertex being non-adjacent with some edge, as all vertices are adjacent to each other. Consider a vertex $u$ in $k_{n}$, this vertex has $(n-1)$ edges incident to it. There are $\frac{n(n-1)}{2}$ edges which means $\frac{n(n-1)}{2}-(n-1)$ edges are not incident on it. Each of these edges are incident on two other vertices in $k_{n}$, but those two vertices are adjacent to $u$ forming a triangle. This is same with all the other non-adjacent edges.

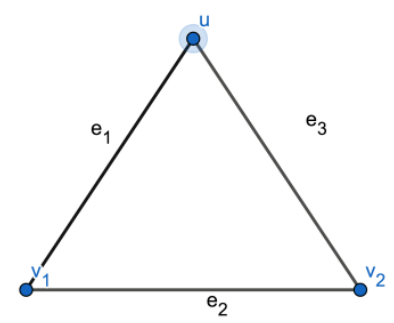

Therefore an edge say $e_{2}$ that is not adjacent to $u$ is incident on two other vertices which are also adjacent to $u$ in $T\left(k_{n}\right)$. Since it's a triangle, the same edge is adjacent to two edges which are incident on $u$, as it's a triangle. Therefore, totally there are 4 vertices that are adjacent to the non-adjacent vertices in $T\left(k_{n}\right)$. Hence the Lemma.

\section{RESULTS AND DISCUSSIONS}

Theorem 4.1: Total graph of a complete graph is Strongly regular graph.

Proof : From the definition of strongly regular graph,

A graph $\mathrm{G}$ of order $n$ is called strongly regular with parameters $(n, k, \lambda, \mu)$ if

- $\quad$ every vertex of $\mathrm{G}$ has degree $\mathrm{k}$,

- if $u$ and $v$ are adjacent vertices of $G$, then the number of common neighbors of $u$ and $v$ is $\lambda$,

- if $u$ and $v$ are non-adjacent vertices of $G$, then the number of common neighbors of $u$ and $v$ is $\mu$

From Lemma 3.1.1, Vertices of the total graph obtained from the complete graph is $\frac{n(n+1)}{2}$.

From Lemma 3.1.2, Total graph of a complete graph is $2(n-1)$ regular.

From Lemma 3.2.1. In a total graph of a complete graph with $n>2$, the number of common neighbours for any two adjacent vertices is $n-1$.

From Lemma 3.2.2. In a total graph of a complete graph with $n>2$, the number of common neighbours for any two nonadjacent vertices is 4 .

Hence, the graph $T\left(k_{n}\right)$ where $n>2$ is strongly regular with parameters $\left(\frac{n(n+1)}{2}, 2(n-1),(n-1), 4\right)$

\subsection{Spectrum and Energy of a total graph obtained from a complete graph}

The set of eigen values obtained by means of the adjacency matrix of graph is called the spectra or spectrum of the graph. The general representation is

$\operatorname{Spec}(G)=\left(\begin{array}{llll}\lambda_{1} & \lambda_{2} & \cdots & \cdots \\ n_{1} & n_{2} & \cdots & \cdots\end{array}\right)$

Total graph of a complete graph is Strongly regular graph from Theorem 4.1. The adjacency matrix of a strongly regular graph has exactly three eigen values. Hence the Total graph of a complete graph also has three eigen values. 


\section{International Advanced Research Journal in Science, Engineering and Technology}

Vol. 6, Issue 3, March 2019

Theorem 4.2: The total graph of a complete graph $k_{n}$ with $n>2$, has spectrum $\left(\begin{array}{ccc}2(n-1) & (n-3) & -2 \\ 1 & n & \frac{(n-2)(n+1)}{2}\end{array}\right)$ And energy $(n-2)(n+1)$.

Proof : The total graph of a complete graph is strongly regular and hence has three eigen values.

One of the eigen values is the regularity with multiplicity 1. From Lemma 3.1.2, one of the eigen value is $2(n-1)$

with multiplicity 1 . The other two eigen values are given by $\frac{(\lambda-\mu) \pm \sqrt{(\lambda-\mu)^{2}+4(\kappa-\mu)}}{2}$ (eq1)

From Lemma 3.1.2 $\mathrm{k}=2(\mathrm{n}-1)$

From Lemma 3.2.1. $\lambda=(n-1)$

From Lemma 3.2.2. $\mu=4$

Substituting these in equation (1) we get $(n-3)$ and -2 as the eigen values.

To find the multiplicities of eigen values,

According to the spectral theorem, adjacency matrix A is diagonalizable. Also traceA $=\sum \lambda_{\mathrm{i}}$

Here trace $\mathrm{A}=0$ so also $\sum \lambda_{\mathrm{i}}=0$

Let ' $a$ ' be the multiplicity of $(n-3)$ and ' $b$ ' be the multiplicity of -2

Therefore, regularity $+b(-2)+a(n-3)=0$

ie, $2(n-1)+b(-2)+a(n-3)=0$

Since sum of multiplicities is equal to the number of vertices

$$
1+a+b=\frac{n(n+1)}{2}
$$

Solving equation 2 and equation 3 , we get

$$
\mathrm{a}=\mathrm{n} \quad \text { and } \quad \mathrm{b}=\frac{(\mathrm{n}-2)(\mathrm{n}+1)}{2}
$$

\section{The Energy of total graph of a complete graph $\mathbf{k}_{\mathbf{n}}$}

The Energy of total graph of a complete graph $\mathrm{k}_{\mathrm{n}}$ with $\mathrm{n}>2$, is given by $\mathrm{E}\left(\mathrm{T}\left(\mathrm{k}_{\mathrm{n}}\right)\right)=\sum_{\mathrm{i}=1}^{\mathrm{n}}\left|\lambda_{\mathrm{i}}\right|$

$$
\begin{aligned}
\mathrm{E}\left(\mathrm{T}\left(\mathrm{k}_{\mathrm{n}}\right)\right) & =2(\mathrm{n}-1)+\mathrm{n}(\mathrm{n}-3)+\frac{(\mathrm{n}-2)(\mathrm{n}+1)}{2}|-2| \\
& =2(\mathrm{n}-2)(\mathrm{n}+1)
\end{aligned}
$$

Hence the theorem.

\subsection{Illustrations}

\subsubsection{Consider the total graph of $K_{3}$}

The adjacency matrix is

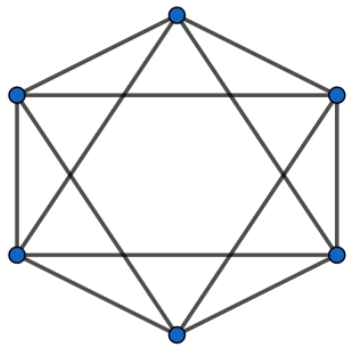

Fig. $1 \mathrm{~T}\left(\mathrm{k}_{3}\right)$,

$$
\mathrm{A}\left(\mathrm{T}\left(\mathrm{k}_{3}\right)\right)=\left(\begin{array}{llllll}
0 & 1 & 1 & 1 & 0 & 1 \\
1 & 0 & 1 & 1 & 1 & 0 \\
1 & 1 & 0 & 0 & 1 & 1 \\
1 & 1 & 0 & 0 & 1 & 1 \\
0 & 1 & 1 & 1 & 0 & 1 \\
1 & 0 & 1 & 1 & 1 & 0
\end{array}\right)
$$

By observation $\mathrm{T}\left(\mathrm{k}_{3}\right)$ has 6 vertices, its 4 regular, $\lambda=2, \mu=4$

When calculated manually, the eigen values are

$$
\begin{aligned}
& \lambda^{3}(\lambda+2)^{2}(\lambda-4)=\mathbf{0} \\
& \lambda=0, \lambda=-2 \text { and } \lambda=4
\end{aligned}
$$


And the spectrum is $\left(\begin{array}{ccc}4 & 0 & -2 \\ 1 & 3 & 2\end{array}\right)$

with energy $\mathrm{E}\left(\mathrm{T}\left(k_{3}\right)\right)=\sum_{i=1}^{n}\left|\lambda_{i}\right|$

$$
=0+|-2|+|-2|+|4|=8
$$

\section{Using the obtained results,}

$\mathrm{T}\left(k_{3}\right)$ is strongly regular with parameters $\left(\frac{n(n+1)}{2}, 2(n-1),(n-1), 4\right)$

Substituting $\mathrm{n}=3$, we get 6vertices, 4-regular, $\lambda=2, \mu=4$

spectrum is given by $\left(\begin{array}{ccc}2(n-1) & (n-3) & -2 \\ 1 & n & \frac{(n-2)(n+1)}{2}\end{array}\right)$

Substituting $n=3$, the spectrum is $\left(\begin{array}{ccc}4 & 0 & -2 \\ 1 & 3 & 2\end{array}\right)$

And the energy is given by $2(n-2)(n+1)=(2)(1)(4)=8$

\subsubsection{Consider the total graph of $K_{4}$}

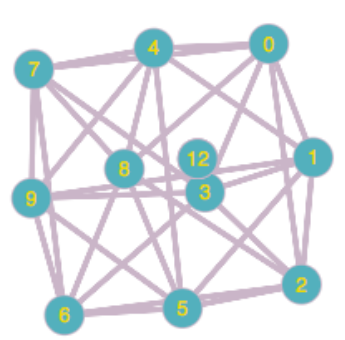

Fig. $2 \mathrm{~T}\left(k_{4}\right)$,

The adjacency matrix is

$$
\mathrm{A}\left(\mathrm{T}\left(k_{4}\right)\right)=\left(\begin{array}{cccccccccc}
0 & 1 & 1 & 1 & 1 & 0 & 0 & 1 & 1 & 0 \\
1 & 0 & 1 & 1 & 1 & 1 & 0 & 0 & 0 & 1 \\
1 & 1 & 0 & 1 & 0 & 1 & 1 & 0 & 1 & 0 \\
1 & 1 & 1 & 0 & 0 & 0 & 1 & 1 & 0 & 1 \\
1 & 1 & 0 & 0 & 0 & 1 & 0 & 1 & 1 & 1 \\
0 & 1 & 1 & 0 & 1 & 0 & 1 & 0 & 1 & 1 \\
0 & 0 & 1 & 1 & 0 & 1 & 0 & 1 & 1 & 1 \\
1 & 0 & 0 & 1 & 1 & 0 & 1 & 0 & 1 & 1 \\
1 & 0 & 1 & 0 & 1 & 1 & 1 & 1 & 0 & 0 \\
0 & 1 & 0 & 1 & 1 & 1 & 1 & 1 & 0 & 0 \\
& & & & & & & & &
\end{array}\right)
$$

By observation $\mathrm{T}\left(k_{4}\right)$ has 10 vertices, its 6 regular, $\lambda=3, \mu=4$

When calculated by conventional method, the eigen values are

$(\lambda+2)^{3}(\lambda-1)^{4}(\lambda-6)=0$

$\lambda=-2, \lambda=1$ and $\lambda=6$

And the spectrum is $\left(\begin{array}{ccc}6 & 1 & -2 \\ 1 & 4 & 3\end{array}\right)$

with energy $\mathrm{E}\left(\mathrm{T}\left(k_{4}\right)\right)=\sum_{i=1}^{n}\left|\lambda_{i}\right|$

$$
=|6|+|1|+|1|+|1|+|1|+|-2|+|-2|+|-2|+|-2|+|-2|=20
$$

\section{Using the obtained results,}

$\mathrm{T}\left(k_{4}\right)$ is strongly regular with parameters $\left(\frac{n(n+1)}{2}, 2(n-1),(n-1), 4\right)$

Substituting $\mathrm{n}=4$, we get 10 vertices, 6-regular, $\lambda=3, \mu=4$

spectrum is given by $\left(\begin{array}{ccc}2(n-1) & (n-3) & -2 \\ 1 & n & \frac{(n-2)(n+1)}{2}\end{array}\right)$ 
Substituting $\mathrm{n}=4$, the spectrum is $\left(\begin{array}{ccc}6 & 1 & -2 \\ 1 & 4 & 5\end{array}\right)$

And the energy is given by $2(n-2)(n+1)=(2)(2)(5)=20$

\subsubsection{Consider the total graph of $K_{5}$}

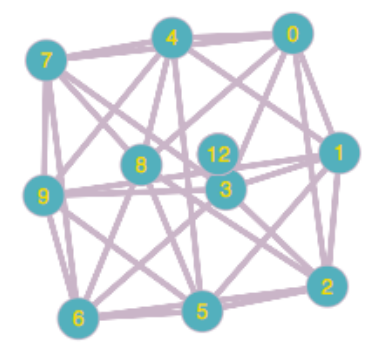

Fig. $3 \mathrm{~T}\left(k_{5}\right)$,

The adjacency matrix of $\mathrm{A}\left(\mathrm{T}\left(k_{5}\right)\right)$ has dimension 15 . Due to large dimensions the eigen values are calculated using MATLAB.

$A=$

By observation $\mathrm{T}\left(k_{5}\right)$ has 15 vertices, its 8 regular, $\lambda=4, \mu=4$

When calculated using MATLAB, the following eigen values were obtained

$$
\begin{gathered}
>\mathrm{e}=\mathrm{eig}(\mathrm{A}) \\
\mathrm{e}= \\
-2.0000 \\
-2.0000 \\
-2.0000 \\
-2.0000 \\
-2.0000 \\
-2.0000 \\
-2.0000 \\
-2.0000 \\
-2.0000 \\
2.0000 \\
2.0000 \\
2.0000 \\
2.0000 \\
2.0000 \\
8.0000
\end{gathered}
$$

Hence the spectrum is $\left(\begin{array}{ccc}8 & 2 & -2 \\ 1 & 5 & 9\end{array}\right)$ with energy $\mathrm{E}\left(\mathrm{T}\left(k_{4}\right)\right)=\sum_{i=1}^{n}\left|\lambda_{i}\right|$

$$
=|8|+|2|+|2|+|2|+|2|+|2|+|-2|+|-2|+|-2|+|-2|+|-2|+|-2|+|-2|+|-2|+|-2|=36
$$

Using the obtained results,

$\mathrm{T}\left(k_{5}\right)$ is strongly regular with parameters $\left(\frac{n(n+1)}{2}, 2(n-1),(n-1), 4\right)$

Substituting $\mathrm{n}=5$, we get 15 vertices, 8 -regular, $\lambda=4, \mu=4$ 


\section{International Advanced Research Journal in Science, Engineering and Technology}

Vol. 6, Issue 3, March 2019

spectrum is given by $\left(\begin{array}{ccc}2(n-1) & (n-3) & -2 \\ 1 & n & \frac{(n-2)(n+1)}{2}\end{array}\right)$

Substituting $\mathrm{n}=5$, the spectrum is $\left(\begin{array}{ccc}8 & 2 & -2 \\ 1 & 5 & 9\end{array}\right)$

And the energy is given by $2(n-2)(n+1)=(2)(3)(6)=36$

Hence the results obtained can be used in calculating the four parameters of total graph of a complete graph. The Energy and spectrum of a total graph of complete graph can be directly calculated using the derived formulas. Whereas the conventional methods become tedious as the number of vertices of graph increases. The three illustrations shown here explains this

\section{CONCLUSION}

The Theorems can be understood as a general formula for finding the four parameters of total graph of a complete graph. Which are $\left(\frac{n(n+1)}{2}, 2(n-1),(n-1), 4\right) \quad$ For $\mathrm{n}>2$. The eigen values, graph spectrum and energy of graph are obtained by graph operations. The graph energy is calculated for most of the graphs, there are many papers published on it. But this paper is on energy of graph obtained by graph operations. The study revealed that the eigen values of total graph of a complete graph $\left(\mathrm{T}\left(\mathrm{K}_{\mathrm{n}}\right)\right)$ for $\mathrm{n}>2$ are ' $2(n-1)$ ' repeated 1 time, ' $(n-3)$ ' repeated ' $\mathrm{n}$ ' times, ' -2 ' repeated $\frac{(n-2)(\mathrm{n}+1)}{2}$ times.

The spectrum of $\left(\mathrm{T}\left(\mathrm{K}_{\mathrm{n}}\right)\right)$ is given by $\left(\begin{array}{ccc}2(\mathrm{n}-1) & (\mathrm{n}-3) & -2 \\ 1 & \mathrm{n} & \frac{(\mathrm{n}-2)(\mathrm{n}+1)}{2}\end{array}\right)$

The energy of $\left(T\left(K_{n}\right)\right)$ is given by $2(n-2)(n+1)$.

\section{REFERENCES}

[1]. Linear Algebra and its applications, 432(2010), 1825-1835

[2]. C. Berge, Theory of graphs and its applications, Dunod, Paris, (1958)

[3]. Gutman, Hyperenergetic and hypo energetic graphs, in: D. Cvetković, I. Gutman (Eds.), Selected Topics on Applications of Graph Spectra, Math. Inst., Belgrade, 2011, pp. 113-135.

[4]. C.A Coulson, on the calculation of the energy in unsaturated hydrocarbon molecules, Proc. Cambridge Phil. Soc, 36(1940), 201-203

[5]. D. F. Anderson and A. Badawi, The total graph of a commutative ring, J. Algebra, 320, (2008), 2706-2719

[6]. I. Gutman and B. Zhou, Laplacian energy of a graph, Linear Algebra Appli, 414 (2006), 29-37

[7]. F. Harary, Graph Theory, Addison Wesley, Reading Mans (1972).

[8]. T. Tamizh Chelvam, S. Raja and I. Gutman, Strongly regular integral circulant graphs and their energies, Bull. Int. Math. Virt. Inst.,2, (2012), $9-16$.

[9]. R. C. Bose, Strongly regular graphs, partial geometries and partially balanced designs, Pacific Journal of Mathematics 13 (1963), no. 2, 389419.

[10]. Chris Godsil and Gordon Royle (2004), Algebraic Graph Theory. New York: Springer-Verlag. ISBN 0-387-95241-1

[11]. G. Indulal and I. Gutman, On the distance spectra of some graphs, Matteratical communication, (2008), 123-131.

[12]. X. Li, Y. Shi, and I. Gutman, Graph Energy, Springer, New York, 2012.

[13]. O. Ore, Theory of Graphs, Aner. Math. Soc. Collog. Publication, 38, Providence (1962)

[14]. I. Gutman and X. Li, Energies of Graphs. Theory and Applications, University of Kragujevac, Kragujevac, Serbia, 2016. 